\title{
Perioperative Predictive Markers for Recurrence of Esophageal Cancer after Esophagectomy
}

\author{
Yoshinori Fujiwara Masaharu Higashida Hisako Kubota Yuko Okamoto \\ Shumei Mineta Shunji Endo Tomio Ueno \\ Department of Digestive Surgery, Kawasaki Medical School, Kurashiki, Japan
}

\section{Keywords \\ Esophageal cancer · Prognostic nutritional index · \\ C-reactive protein · Cancer progression · Prognosis}

\begin{abstract}
Introduction: We studied whether perioperative nutritional, immunological factors or postoperative inflammatory responses predicted esophageal cancer (EC) progression and prognosis in patients who received esophagectomies. Methods: We evaluated preoperative prognostic nutritional index (PNI), BMI, neutrophil-to-lymphocyte ratio (NLR), intraoperative blood loss, postoperative C-reactive protein (CRP) max, recurrence-free survival (RFS), and overall survival (OS) in 111 patients with pStage I-IV squamous cell EC who received esophagectomies. Optimal cutoff values for each continuous parameter were determined by receiver operating characteristic curves and Youden indices. Univariate and multivariate Cox analyses were used to derive independent prognostic factors. Propensity score matching using inverse probability of treatment weighting was used in groups divided by Youden indices, as appropriate. Results: Cutoff values of continuous variables were NLR: 2.27, PNI: 44.2, blood loss: $159 \mathrm{~mL}$, and CRPmax: $21.7 \mathrm{mg} / \mathrm{dL}$. In multivariate analy-
\end{abstract}

karger@karger.com www.karger.com/gat

Karger $\frac{1}{\%}$

GOPEN ACCESS
(C) 2021 The Author(s)

Published by S. Karger AG, Basel

This article is licensed under the Creative Commons AttributionNonCommercial-NoDerivatives 4.0 International License (CC BYNC-ND) (http://www.karger.com/Services/OpenAccessLicense) Usage and distribution for commercial purposes as well as any distribution of modified material requires written permission. ses, PNI, CRPmax, and intraoperative blood loss were independent prognostic factors for OS and RFS. Among patients with stage II-IV disease, low PNI was associated with shorter RFS. Postoperative respiratory complications were associated with both higher CRP and shorter RFS. Discussion/Conclusions: Low preoperative PNI and high postoperative inflammatory response were associated with postoperative EC progression after esophagectomy. Preoperative nutritional interventions or suppression of postoperative inflammatory response, including respiratory complications, may improve patient prognosis.

(c) 2021 The Author(s)

Published by S. Karger AG, Basel

\section{Introduction}

Esophageal cancer (EC) is the seventh most common cancer worldwide [1]. Its 2 most common histologic subtypes are adenocarcinoma and squamous cell carcinoma (SCC). SCC is more common in Asian countries, where most ECs are of the SCC subtype; only $4.5 \%$ of ECs in Japan were adenocarcinomas [2]. Surgery is the standard treatment for patients with locoregionally confined EC. Despite the availability of curative surgery, EC has a high 
rate of recurrence [3-5]. Two-thirds of patients with EC have advanced disease at the time of admission; most patients with advanced EC complain of dysphagia, which leads to weight loss and malnutrition $[2,6]$. In addition, host-tumor interactions affect the nutritional status and immune system in cancer patients [7], which may exacerbate weight loss and malnutrition in patients with EC. Nutritional and immunological statuses are shown to be significant predictors of cancer progression and survival [811]. Preoperative nutritional and/or immunological factors, including BMI, albumin, prognostic nutritional index (PNI), and neutrophil-to-lymphocyte ratio (NLR), were evaluated with respect to progression in many types of cancer, including EC [12-17]. C-reactive protein (CRP) is an acute-phase serum protein and a well-established inflammatory marker; at high concentrations, it is associated with high mortality in solid tumors, including gastrointestinal malignancies [18]. Some studies have investigated postoperative CRP levels and EC progression and prognosis after esophagectomy [19-21], but each study used different methods to measure CRP. Whether preoperative nutritional or immunological factors or postoperative inflammatory reactions predict EC progression after esophagectomy is unclear. In the present study, we retrospectively evaluated nutritional and immunological factors before EC surgery, inflammatory responses after surgery, and the relationships between cancer progression and these parameters, using multivariate analysis.

\section{Materials and Methods}

This retrospective study included 111 consecutive patients with biopsy-proven invasive esophageal SCC and no history of previous treatment, who underwent esophagectomies with 2- or 3-field lymphadenectomies, at the Kawasaki Medical School Hospital between January 2010 and June 2019. Tumor staging was based on the UICC classification of malignant tumors (8th edition) [22]. We excluded patients with $\mathrm{R} 1 / 2$ resections and those who received preoperative chemotherapy or chemoradiotherapy. This study was approved by the Kawasaki Medical School Institutional Review Board (Authorization No. 3382). Details of this study are described on the Kawasaki Medical School home page (https://h.kawasakim.ac.jp/cgi-image/3644/3644_njbGzmAGzJKXvLIXPmMBEPmYEfwPTbPQOlVqmObuLvggagkgbp.pdf). Data were collected from patients' medical records. All patients were followed up regularly until April 2020 or until death. Informed consent was obtained from all patients.

Nutritional, Immunological, and Inflammatory Response

Assessment

Peripheral blood samples were collected within 1-4 weeks before surgery. Preoperative PNI was calculated as $(10 \times$ albumin $[\mathrm{g} /$ $\mathrm{dL}])+\left(0.005 \times\right.$ peripheral lymphocyte counts $\left./ \mathrm{mm}^{3}\right)$ [23]. Preop- erative NLR was defined as absolute neutrophil count divided by absolute lymphocyte count [24]. Preoperative BMI was defined as $\mathrm{kg} / \mathrm{m}^{2}$ [25]. Other preoperative nutritional indices, including serum albumin ( $\mathrm{mg} / \mathrm{dL})$, were also evaluated. The systemic postoperative inflammatory response was evaluated based on serum CRP level (mg/dL), which was measured on postoperative days $1,2,3$, 5 , and 7 and thereafter as necessary. The highest CRP level (CRP$\max$ ) was considered to be a postoperative inflammatory response. We also evaluated the postoperative $\mathrm{CRP} /$ albumin ratio, which was defined as the ratio of the postoperative CRP level divided by the serum albumin level, at the highest CRP value on the postoperative day.

\section{Surgery and Associated Factors}

Three surgical procedures were performed. Ivor Lewis esophagectomies with 2- or 3-field lymph node dissections were performed in the period through 2013. Esophageal reconstructions were performed using a gastric tube in the retrosternal or posterior mediastinal root. This procedure was changed to a thoracoscopic-and laparoscopic-assisted approach after 2013. Transhiatal esophagectomy with middle, lower mediastinal, and abdominal lymphadenectomy was also performed for patients with relatively early-stage EC. Postoperative complications of Clavien-Dindo classification grade 2 or higher were included in our analysis [26]. Intraoperative blood loss, operation time, and length of hospital stay were also evaluated.

\section{Statistical Analysis}

We used the $\chi^{2}$ or Fisher's exact $t$ test for categorical variables and the Mann-Whitney $U$ test for continuous variables. Overall survival (OS) was defined as the time between surgery and death or final available information pertaining to vital status. Recurrence-free survival (RFS) was defined as the time between surgery and cancer recurrence, death, or last available information pertaining to vital status. Optimal cutoff values of variables were used for time-dependent receiver operating characteristic curves for 3 -year OS. The Youden index was used to determine optimal threshold values for preoperative PNI, BMI, NLR, and albumin and postoperative CRPmax, CRP/albumin ratio, and intraoperative blood loss. We used median values as the cutoff values for operation time and hospital stay (days). When each group was divided by the Youden index, including those with a small number of patients or confounding variables, we calculated propensity scores, using a multivariable logistic regression model, with age, sex, cancer site, pTNM stage, lymph node metastasis, operative procedure, tumor length, and depth of tumor invasion (pT) as independent variables. We used the propensity scores to match each group, using the inverse probability of treatment weighting (IPTW) method. Using this method, we performed univariate analyses for OS and RFS in 7 parameters including preoperative PNI, BMI, albumin (mg/dL), NLR, and intraoperative blood loss and postoperative CRP/albumin ratio and CRPmax. Cox proportional hazard models for OS and RFS were used to identify independent prognostic factors. Factors in the Cox model were selected with the following criteria: (a) the number of explanatory variables should be approximately $1 / 10$ of the number of event occurrences, (b) we did not enter factors that were dependent on each other, (c) useful independent prognostic factors were selected from previously published data, and (d) we included factors for which $p<0.2$ in the univariate analysis. Kaplan-Meier curves of
88

Gastrointest Tumors 2021;8:87-95 DOI: $10.1159 / 000513961$
Fujiwara/Higashida/Kubota/Okamoto/ Mineta/Endo/Ueno 
Table 1. Clinical characteristics in this study

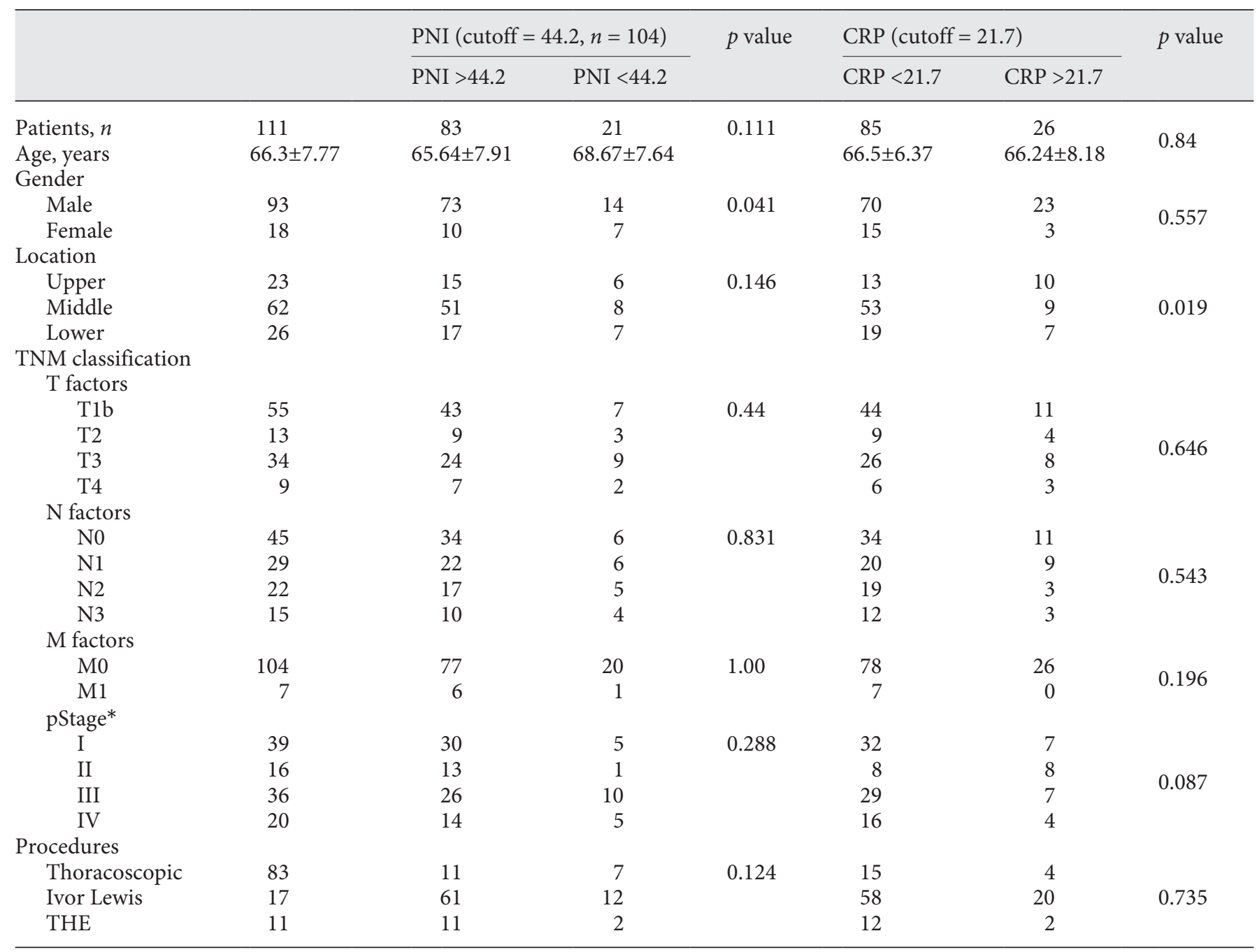

PNI, prognostic nutritional index; CRP, C-reactive protein; THE, transhiatal esophagectomy. * UICC TNM 8th edition.

estimated RFS were generated and compared between the groups, using log-rank tests. $p<0.05$ was considered significant. Statistical analyses were performed using JMP (version 14; SAS, Tokyo, Japan), Stata (Version14; LightStone, Tokyo, Japan), and R version 3.1.1 (R Project for Statistical Computing; Vienna, Austria).

\section{Results}

\section{Patient Characteristics}

We evaluated 111 patients with squamous cell EC in this study (Table 1). Their 3-year OS and RFS were 58.8 and $57.5 \%$, respectively. There were 44 events (death) between observation periods. Mean follow-up time was
1,233 days (range: 111-3,256 days). Cutoff values were PNI: 44.2 (false positive: 0.891 , true positive: 0.333 , and area under the curve: 0.585 ) and CRPmax: $21.7 \mathrm{mg} / \mathrm{dL}$ (false positive: 0.809 , true positive: 0.326 , and area under the curve: 0.589). Clinical factors related to postoperative CRPmax or preoperative PNI divided by the cutoff values are shown in Table 1. The PNI groups had 104 patients because 7 patients did not have preoperative lymphocyte counts. Table 2 shows univariate analysis of prognostic factors for OS and RFS, with cutoff continuous variables, including PNI and CRPmax. Preoperative PNI, albumin and intraoperative blood loss, pTNM, and tumor location were prognostic factors for OS and RFS. Postoperative CRPmax was an independent prognostic 
Table 2. Univariate analysis for OS and RFS in the present study

\begin{tabular}{|c|c|c|c|c|c|c|c|}
\hline \multirow[t]{2}{*}{ Covariants } & \multirow{2}{*}{$\begin{array}{l}\text { Cutoff value } \\
(\mathrm{FP}, \mathrm{TP})\end{array}$} & \multirow[t]{2}{*}{$\mathrm{HR}$} & \multicolumn{2}{|l|}{ RFS } & \multirow[t]{2}{*}{$\mathrm{HR}$} & \multicolumn{2}{|l|}{ OS } \\
\hline & & & $95 \% \mathrm{CI}$ & $p$ value & & $95 \% \mathrm{CI}$ & $p$ value \\
\hline Age & & 1.068 & $0.819-1.394$ & 0.627 & 1.117 & $0.855-1.461$ & 0.417 \\
\hline Gender & & 0.969 & $0.432-2.176$ & 0.94 & 0.859 & $0.362-2.036$ & 0.729 \\
\hline $\mathrm{pT}$ & & 1.622 & $1.247-2.109$ & $0.001 *$ & 1.521 & $1.159-1.995$ & $0.003 *$ \\
\hline $\mathrm{pN}$ & & 1.658 & $1.270-2.165$ & $0.001 *$ & 1.674 & $1.265-2.214$ & $0.001 *$ \\
\hline $\mathrm{pM}$ & & 1.477 & $0.528-4.133$ & 0.457 & 1.333 & $0.475-3.741$ & 0.585 \\
\hline pTNM & & 1.829 & $1.378-2.428$ & $0.001 *$ & 1.726 & $1.296-2.300$ & $0.001 *$ \\
\hline Tumor location & & 0.467 & $0.292-0.747$ & $0.002 *$ & 0.394 & $0.243-0.637$ & $0.001 *$ \\
\hline Operative procedures & & 0.685 & $0.392-1.197$ & 0.184 & 0.837 & $0.491-1.429$ & 0.515 \\
\hline Preoperative PNI & $44.2(0.891,0.333)$ & 0.292 & $0.155-0.552$ & $0.001 *$ & 0.315 & $0.164-0.606$ & $0.001 *$ \\
\hline Preoperative albumin, g/dL & $3.5(0.897,0.279)$ & 0.459 & $0.251-0.839$ & $0.011 *$ & 0.471 & $0.254-0.876$ & $0.017 *$ \\
\hline NLR & $2.272(0.484,0.600)$ & 0.84 & $0.459-1.536$ & 0.571 & 0.716 & $0.382-1.342$ & 0.297 \\
\hline BMI, $\mathrm{kg} / \mathrm{m}^{2}$ & $21.4(0.441,0.698)$ & 0.669 & $0.354-1.263$ & 0.216 & 0.686 & $0.364-1.295$ & 0.246 \\
\hline Blood loss, $g$ & $159(0.515,0.767)$ & 2.549 & $1.313-4.946$ & $0.006 *$ & 2.467 & $1.242-4.897$ & $0.01 *$ \\
\hline CRPmax & $21.7(0.809,0.326)$ & 1.944 & $1.047-3.608$ & $0.0321^{*}$ & 1.735 & $0.9023-3.337$ & 0.0942 \\
\hline $\mathrm{CRP} /$ albumin ratio & $8.512(0.735,0.395)$ & 1.702 & $0.939-3.084$ & 0.076 & 1.726 & $0.933-3.193$ & 0.081 \\
\hline Operation time & $360 \min ($ median) & 1.369 & $0.735-2.550$ & 0.322 & 1.26 & $0.690-2.304$ & 4.522 \\
\hline Hospital stay & 42 days (median) & 1.161 & $0.629-2.144$ & 0.663 & 1.32 & $0.724-2.409$ & 0.365 \\
\hline Postoperative complications & & 1.129 & $0.625-2.041$ & 0.625 & 1.056 & $0.583-1.941$ & 0.855 \\
\hline
\end{tabular}

OS, overall survival; RFS, recurrence-free survival; FP, false positive TP, true positive; PNI, prognostic nutritional index; CRP, Creactive protein; NLR, neutrophil-to-lymphocyte ratio. * Statistically significant.

Table 3. Univariate analysis for RFS and OS of advanced EC patients after IPTW

\begin{tabular}{lllllll}
\hline Covariants & HR & $\begin{array}{l}\text { OS after IPTW } \\
95 \% \text { CI }\end{array}$ & $p$ value & HR & $\begin{array}{l}\text { RFS after IPTW } \\
95 \% \text { CI }\end{array}$ & $p$ value \\
\hline PNI & 0.391 & $0.177-0.863$ & $0.02^{*}$ & 0.36 & $0.178-0.729$ & $0.005^{*}$ \\
CRPmax & 1.507 & $0.760-2.988$ & 0.24 & 1.841 & $1.014-3.342$ & $0.045^{*}$ \\
NLR & 0.878 & $0.460-1.674$ & 0.694 & 0.913 & $0.496-1.679$ & 0.771 \\
Blood loss & 1.528 & $0.757-3.085$ & 0.236 & 1.479 & $0.769-2.842$ & 0.24 \\
BMI & 0.767 & $0.393-1.496$ & 0.437 & 0.698 & $0.361-1.347$ & 0.284 \\
Albumin (preoperative) & 0.502 & $0.253-0.995$ & $0.048^{*}$ & 0.496 & $0.263-0.935$ & $0.03^{*}$ \\
CRP/albumin ratio & 1.35 & $0.713-2.558$ & 0.356 & 1.326 & $0.713-2.465$ & 0.372 \\
\hline
\end{tabular}

OS, overall survival; RFS, recurrence-free survival; EC, esophageal cancer; IPTW, inverse probability of treatment weighting; PNI, prognostic nutritional index; CRP, C-reactive protein; NLR, neutrophil-to-lymphocyte ratio. * Statistically significant.

factor for RFS, but not for OS. Postoperative complications were not associated with cancer recurrence and prognosis. Univariate analyses of prognostic factors after IPTW propensity scoring are shown in Table 3. Preoperative PNI was an independent prognostic factor for both OS and RFS. CRPmax was an independent prognostic factor for PFS, but not OS. Intraoperative blood loss was not a prognostic factor for either OS or RFS.
When patients were divided by the cutoff value in to high and low PNI groups, their 3-year RFS rates after IPTW significantly differed (64.5 vs. $36.18 \%$; $p<0.05$; shown in Fig. 1). The 3 -year RFS rates of the low and high CRP groups after IPTW were also significantly different (62.49 vs. $36.18 \%$; $p<0.05$; shown in Fig. 2 ). Among patients with pStage II-IV EC, the high PNI group had better OS and RFS than did the low PNI group $(p<0.01)$;
90

Gastrointest Tumors 2021;8:87-95 DOI: $10.1159 / 000513961$
Fujiwara/Higashida/Kubota/Okamoto/ Mineta/Endo/Ueno 
Table 4. Univariate analysis for OS and RFS between low and high PNI groups after IPTW in different stages

\begin{tabular}{|c|c|c|c|c|c|c|}
\hline \multirow[t]{2}{*}{ Covariants } & \multicolumn{3}{|c|}{ For OS } & \multicolumn{3}{|c|}{ For RFS } \\
\hline & HR & $95 \% \mathrm{CI}$ & $p$ value & HR & $95 \% \mathrm{CI}$ & $p$ value \\
\hline \multicolumn{7}{|l|}{ pStage I } \\
\hline $\begin{array}{l}\text { High versus low PNI } \\
\text { pStage II-IV }\end{array}$ & 0.41 & $0.326-51.66$ & 0.274 & 2.939 & $0.281-30.74$ & 0.368 \\
\hline High versus low PNI & 0.233 & $0.125-0.432$ & $0.001^{*}$ & 0.24 & $0.130-0.440$ & $0.001^{*}$ \\
\hline
\end{tabular}

OS, overall survival; RFS, recurrence-free survival; IPTW, inverse probability of treatment weighting; PNI, prognostic nutritional index. * Statistically significant.

Table 5. Multivariate Cox analysis for RFS and OS in EC patients

\begin{tabular}{|c|c|c|c|c|c|c|}
\hline & \multicolumn{2}{|c|}{ For RFS } & \multirow[t]{2}{*}{$p$ value } & \multicolumn{2}{|c|}{ For OS } & \multirow[t]{2}{*}{$p$ value } \\
\hline & HR & $95 \%$ CI & & HR & $95 \%$ CI & \\
\hline Age & 0.9123 & $0.498-1.670$ & 0.766 & 0.924 & $0.489-1.743$ & 0.807 \\
\hline pTNM & 1.745 & $1.305-2.334$ & $0.001^{*}$ & 1.757 & $1.281-2.410$ & $0.001^{*}$ \\
\hline PNI & 0.258 & $0.133-0.498$ & $0.001^{*}$ & 0.292 & $0.145-0.588$ & $0.001^{*}$ \\
\hline CRPmax & 2.432 & $1.226-4.823$ & $0.01^{*}$ & 2.079 & $1.010-4.282$ & $0.047^{*}$ \\
\hline Blood loss & 1.92 & $1.001-3.683$ & $0.049^{*}$ & 2.264 & $1.116-4.589$ & $0.023^{*}$ \\
\hline
\end{tabular}

OS, overall survival; RFS, recurrence-free survival; EC, esophageal cancer; PNI, prognostic nutritional index; CRP, C-reactive protein. * Statistically significant.

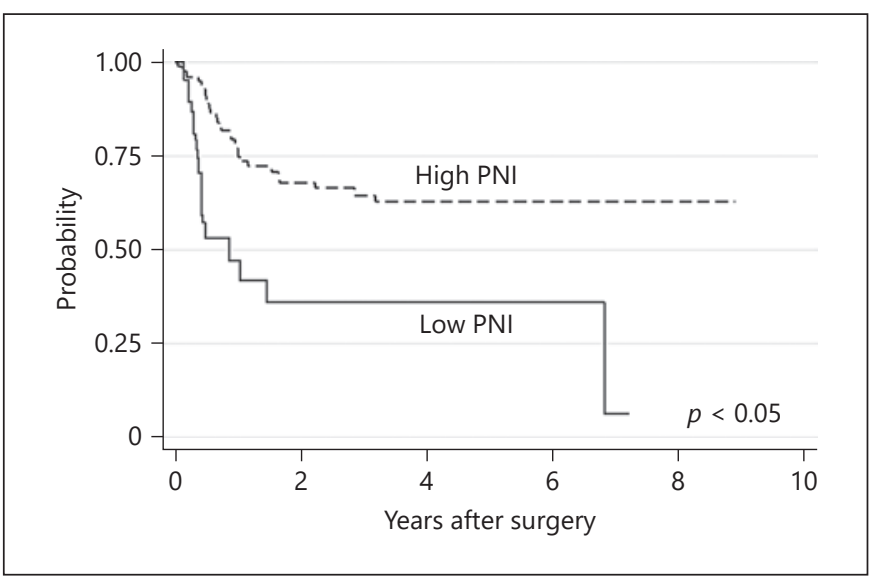

Fig. 1. Kaplan-Meier curves for RFS significantly differed between the high and low PNI groups $(p<0.05)$. RFS, recurrence-free survival; PNI, prognostic nutritional index.

however, among pStage I patients, OS and RFS did not significantly differ between the high and low PNI groups after IPTW (Table 4). PNI, postoperative CRPmax, intraoperative blood loss, and pTNM stage were indepen-

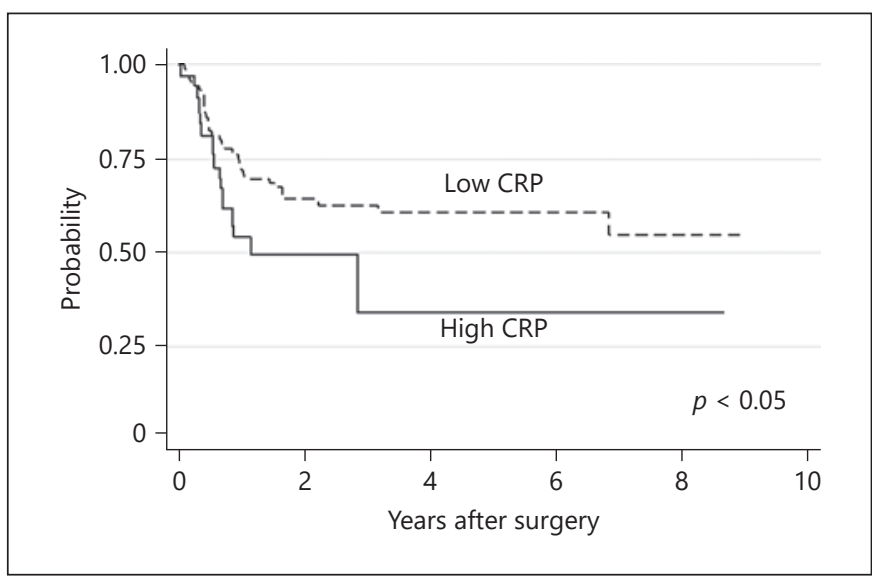

Fig. 2. Kaplan-Meier curves for RFS significantly differed between the high and low CRP groups $(p<0.05)$. RFS, recurrence-free survival; CRP, C-reactive protein.

dent prognostic factors for RFS and OS (Table 5). Postoperative complications (Clavien-Dindo $\geq 2$ ) are shown in Table 6. Fifty patients suffered postoperative complications, including $25(22.5 \%, 25 / 111)$ who suffered re- 
Table 6. Postoperative complications in this study, Clavien-Dindo $\geq 2$

\begin{tabular}{lc}
\hline Complications & Incidence, $n / N(\%)$ \\
\hline Anastomotic leakages & $9 / 111(8.1)$ \\
Recurrent nerve palsy & $11 / 111(9.9)$ \\
Pneumonia & $17 / 111(19.9)$ \\
Pleural effusion & $8 / 111(7.2)$ \\
SSI & $12 / 111(10.71)$ \\
Others & $5 / 111(4.5)$ \\
\hline
\end{tabular}

Others: chylothorax, ileus, peritonitis (2 patients), and abdominal abscess. Duplicate cases were included. SSI, surgical site infection.

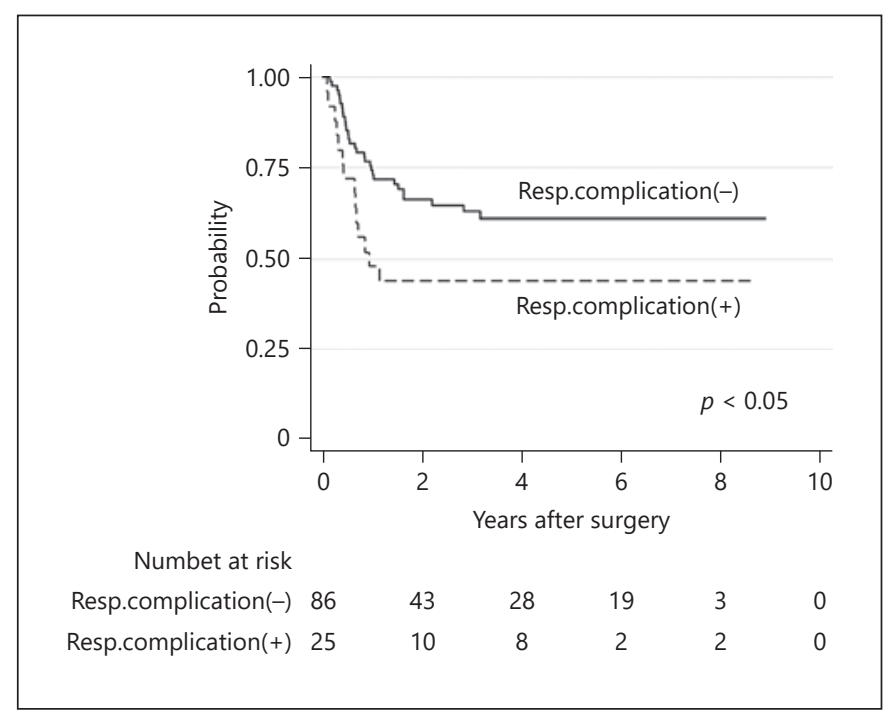

Fig. 3. Kaplan-Meier curves for RFS were significantly worse in patients with postoperative respiratory complications than in patients without postoperative respiratory complications $(p<0.05)$. RFS, recurrence-free survival.

spiratory complications (pneumonia, pleural effusion, and chylothorax). The postoperative complication group had a higher median CRP level $(18.41 \mathrm{mg} / \mathrm{dL})$ than did the no-complication group $(16.10 \mathrm{mg} / \mathrm{dL})$ but not significantly so $(p=0.062)$. However, the median value of CRP in the respiratory complication group $(20.92 \mathrm{mg} / \mathrm{dL})$ was significantly greater than that of the no-complication group $(16.79 \mathrm{mg} / \mathrm{dL} ; p<0.05)$. Figure 3 shows RFS among patients with and without postoperative respiratory complications. Three-year RFS was $63.5 \%$ without respiratory complications and $44 \%$ with complications $(p<0.05)$.

\section{Discussion}

The present study retrospectively evaluated perioperative nutritional and immunological parameters and inflammatory response after esophagectomy and their effects, if any, on cancer progression and prognosis in patients with squamous cell EC. In multivariate analysis, PNI, CRPmax, and intraoperative blood loss were independent prognostic factors for OS and RFS. PNI was first introduced by Onodera et al. [23] in 1984, as an indicator of postoperative complications. A recent meta-analysis showed that lower PNI was correlated with unfavorable prognosis in patients with EC $[16,17,27]$. However, this meta-analysis included patients who had received neoadjuvant treatment and/or chemoradiotherapy. We excluded such patients in the present study to avoid biasing prognostic factors by inserting these factors in multivariate analysis. We found 7 articles on preoperative PNI and prognosis after EC surgery in patients who did not receive neoadjuvant treatment [28-34]. The findings of these studies are controversial; only 2 of 7 studies showed a favorable survival in the high preoperative PNI group, while the other studies arrived at conflicting results. One reason for this difference might be that patients with all stages of cancer were included, and subgroup analyses were not performed in these studies. Therefore, we analyzed the association between PNI and prognosis in EC patients in various stages of cancer (pStage I vs. II-IV) using the IPTW method of propensity scoring. Survival by patients with pStage I EC was similar in the low and high PNI groups, probably because most stage I patients are asymptomatic and few have malnutrition, which generally develops as the cancer progresses [6]. Poor prognosis in the low PNI group was seen in patients with more advanced EC. Therefore, preoperative nutritional intervention may improve the prognosis of advanced EC patients. However, there are some problems with preoperative nutritional intervention in advanced cancer. McMillan et al. [35] reported that systemic inflammatory response plays a major role in the progressive nutritional and functional decline of patients with cancer. Based on this finding, Crumley et al. [36] reported that low albumin concentrations were associated with low survival in patients with gastric cancer, and it was dependent on the presence of a systemic inflammatory response from tumor. Furthermore, Ligthart-Melis et al. [37] reported that the benefits of preoperative nutritional support in patients with EC were found mainly in neoadjuvant settings. Thus, preoperative nutritional support in patients with EC is presumed to be effective only for limited pop-
Fujiwara/Higashida/Kubota/Okamoto/ Mineta/Endo/Ueno 
ulations, and it is a possibility that improved preoperative nutritional status may improve the survival in these patients. We hope that further examinations or clinical study including nutritional intervention in advanced EC might be planned in the future.

We found CRP to be an independent prognostic factor for RFS and OS. CRP is an indicator of postoperative inflammatory responses; it is produced by the liver when IL-6 is elevated $[38,39]$ and is easily measurable in clinical practice. Hirai et al. [40] reported that excessive surgical stress aggravates liver metastasis in rat laparotomy and/or thoracotomy models. Postoperative surgery wound fluid obtained from breast cancer patients promoted breast cancer cell proliferation; this fluid contains a large number of inflammatory cytokines [41]. Thus, experimental studies demonstrated that inflammatory cytokines or excessive surgical stress contributes to cancer progression. Postoperative inflammatory cytokines may have caused cancer progression in this study. High preoperative CRP level was associated with cancer progression and poor survival in patients with EC [42-44]. Several studies have demonstrated that postoperative CRP level is associated with EC prognosis, despite the differences in their methods and timing of CRP evaluation [19-21]. We measured postoperative serum CRP levels as CRPmax, based on a report by Saito et al. [45]; however, we do not know whether this method is optimal and are looking forward to further investigations. In this study, we evaluated the associations among postoperative complications after esophagectomy, CRP levels, and cancer progression. Patients with postoperative complications tended to have higher CRPmax levels, but this was not associated with the cancer prognosis (shown in Table 2). CRPmax was significantly higher in patients with respiratory complications. Although we found no relationship between general postoperative complications and prognosis, the RFS of patients whose complications were specifically respiratory was significantly worse than for patients with no complications. Reportedly, IL-6 levels are significantly elevated by acute lung injury or pneumonia after esophagectomy [46, 47]. Based on these reports, we believe that postoperative respiratory complications might lead to marked CRP elevations, and postoperative inflammatory cytokines might activate cancer cells at the microscopic level to cause cancer recurrence and metastasis. Therefore, reducing postoperative respiratory complications might be necessary to improve prognosis in patients with EC.

Blood loss during surgery is reportedly associated with cancer recurrence in patients with gastric cancer who undergo radical surgery $[48,49]$. Komatsu et al. [50] report- ed that intraoperative blood loss during EC surgery was a prognostic factor in multivariate analysis; however, the cutoff value of intraoperative blood loss was $510 \mathrm{~mL}$, which is larger than the value in our study. Because blood loss was not an independent prognostic factor for RFS and OS after IPTW in this study, further study might be necessary. Nevertheless, efforts to reduce intraoperative blood loss may help improve the prognosis of patients with EC. Surgeons should always keep in mind the development of surgical procedures that reduce intraoperative bleeding and prevent postoperative complications.

\section{Conclusions}

PNI is an independent prognostic indicator for patients with advanced EC who undergo esophagectomy. This is the first study to evaluate postoperative CRP, especially its maximum values; we found that high CRP level is associated with EC progression. Interventions by nutritional support teams to improve preoperative nutritional status and suppression of postoperative inflammatory response might improve the prognosis of EC patients after esophagectomy.

\section{Acknowledgements}

We thank Miss Saki and Mrs. Kimura for data collection. We also thank Edanz Group (https://en-author-services.edanzgroup. $\mathrm{com} / \mathrm{ac}$ ) for editing a draft of the manuscript.

\section{Statement of Ethics}

All procedures were followed in accordance with the ethical standards of the responsible committee on human experimentation (institutional and national) and with the Helsinki Declaration of 1964 and later versions. Informed consent or substitute for it was obtained from all patients for being included in the study. All study participants provided informed consent, and the study design was approved by an ethics review board (Authorization No. 3382, Kawasaki Medical School).

\section{Conflict of Interest Statement}

The authors declare that they have no conflicts of interest.

\section{Funding Sources}

This study received no funding. 


\section{Author Contributions}

Y.F. was involved in data analysis, formation of manuscript concept, and drafting the manuscript; S.E. revised the manuscript of important issue and statistical suggestions; M.H. and H.K. were involved in data collection and auxiliary analysis. S.M. and Y.O. assisted with table and figure formation. T.U. agrees to be accountable for all aspects of this work and permission for submission.

\section{References}

1 Bray F, Ferlay J, Soerjomataram I, Siegel RL, Torre LA, Jemal A. Global cancer statistics 2018: GLOBOCAN estimates of incidence and mortality worldwide for 36 cancers in 185 countries. CA Cancer J Clin. 2018 Nov;68(6): 394-424.

2 Tachimori Y, Ozawa S, Ozawa S, Numasaki $\mathrm{H}$, Ishihara $\mathrm{R}$, Matsubara $\mathrm{H}$, et al. Comprehensive registry of esophageal cancer in Japan, 2012. Esophagus. 2019 Jul;16(3):221-45.

3 Hulscher JB, van Sandick JW, Tijssen JG, Obertop H, van Lanschot JJ. The recurrence pattern of esophageal carcinoma after transhiatal resection. J Am Coll Surg. 2000 Aug; 191(2):143-8

4 Mariette C, Balon JM, Piessen G, Fabre S, Van Seuningen I, Triboulet JP. Pattern of recurrence following complete resection of esophageal carcinoma and factors predictive of recurrent disease. Cancer. 2003 Apr 1;97(7): 1616-23.

5 Nakagawa S, Kanda T, Kosugi S, Ohashi M, Suzuki T, Hatakeyama K. Recurrence pattern of squamous cell carcinoma of the thoracic esophagus after extended radical esophagectomy with three-field lymphadenectomy. J Am Coll Surg. 2004 Feb;198(2):205-11.

6 Esophageal cancer. In: Diseases and Conditions. Patient Care and Health Information. 2018. Available from: https://eur02.safelinks. protection.outlook.com/?url= https\%3A\%2F\%2Fwww.mayoclinic. org\%2Fdiseases-conditions\%2Fesophagealcancer $\% 2$ Fsymptoms-causes $\% 2$ Fsyc$20356084 \&$ amp; data $=04 \% 7$ C $01 \% 7 \mathrm{Ca}$. schlenker\%40karger.com\%7C461ade56003c4b8b3bfc08d8e8d1447c\%7C69e7eb606e904 a0590b15b8d6d697087\%7C0\%7C0\%7C637$515332805129984 \% 7$ CUnknown\%7CTWFpbGZsb3d8eyJWIjoiMC4wLjAwMDAiLCJQIjoiV2luMzIiLCJBTiI6Ik1haWwiL CJXVCI6Mn0\%3D\%7C1000\&amp;sdata $=x t f$ hzD\%2FrUC2CwGMn1PIr1xiLSx\%2FboPA Hw2BDEfIcLtE\%3D\&amp;reserved=0

7 Hanahan D, Weinberg RA. The hallmarks of cancer. Cell. 2000 Jan 7;100(1):57-70.

8 Mainous MR, Deitch EA. Nutrition and infection. Surg Clin North Am. 1994 Jun;74(3): 659-76.

9 Schwegler I, von Holzen A, Gutzwiller JP, Schlumpf R, Mühlebach S, Stanga Z. Nutritional risk is a clinical predictor of postoperative mortality and morbidity in surgery for colorectal cancer. Br J Surg. 2010 Jan;97(1):92-7.

10 Di Caro G, Marchesi F, Laghi L, Grizzi F. Immune cells: plastic players along colorectal cancer progression. J Cell Mol Med. 2013 Sep; 17(9):1088-95.
11 Sylman JL, Mitrugno A, Atallah M, Tormoen GW, Shatzel JJ, Tassi Yunga S, et al. The predictive value of inflammation-related peripheral blood measurements in cancer staging and prognosis. Front Oncol. 2018;8:78.

12 Smith M, Zhou M, Whitlock G, Yang G, Offer A, Hui G, et al. Esophageal cancer and body mass index: results from a prospective study of 220,000 men in China and a meta-analysis of published studies. Int J Cancer. 2008 Apr 1; 122(7):1604-10.

13 Mei Z, Shi L, Wang B, Yang J, Xiao Z, Du P, et al. Prognostic role of pretreatment blood neutrophil-to-lymphocyte ratio in advanced cancer survivors: a systematic review and meta-analysis of 66 cohort studies. Cancer Treat Rev. 2017 Jul;58:1-13.

14 Sun L, Zhu Y, Qian Q, Tang L. Body mass index and prognosis of breast cancer: an analysis by menstruation status when breast cancer diagnosis. Medicine. 2018 Jun;97(26):e11220.

15 Gu WS, Fang WZ, Liu CY, Pan KY, Ding R, $\mathrm{Li} \mathrm{XH}$, et al. Prognostic significance of combined pretreatment body mass index (BMI) and BMI loss in patients with esophageal cancer. Cancer Manag Res. 2019;11:3029-41.

16 Li P, Wang X, Lai Y, Zhou K, Tang Y, Che G. The prognostic value of pre-treatment prognostic nutritional index in esophageal squamous cell carcinoma: a meta-analysis. Medicine. 2019 May;98(22):e15280.

17 Xue Y, Zhou X, Xue L, Zhou R, Luo J. The role of pretreatment prognostic nutritional index in esophageal cancer: a meta-analysis. J Cell Physiol. 2019 Nov;234(11):19655-62.

18 Shrotriya S, Walsh D, Bennani-Baiti N, Thomas S, Lorton C. C-reactive protein is an important biomarker for prognosis tumor recurrence and treatment response in adult solid tumors: a systematic review. PLoS One. 2015;10(12):e0143080.

19 Matsuda S, Takeuchi H, Kawakubo H, Fukuda K, Nakamura R, Takahashi T, et al. Correlation between intense postoperative inflammatory response and survival of esophageal cancer patients who underwent transthoracic esophagectomy. Ann Surg Oncol. 2015 Dec;22(13):4453-60.

20 Ibuki Y, Hamai Y, Hihara J, Emi M, Taomoto J, Furukawa T, et al. Role of postoperative Creactive protein levels in predicting prognosis after surgical treatment of esophageal cancer. World J Surg. 2017 Jun;41(6):1558-65.
21 Katsurahara K, Shiozaki A, Fujiwara H, Konishi H, Kudou M, Shoda K, et al. Relationship between postoperative CRP and prognosis in thoracic esophageal squamous cell carcinoma. Anticancer Res. 2018 Nov; 38(11): 6513-8.

22 James D.Brierley MKGCW. UICC: TNM classification of malignant tumors. 8th ed. Kanehara, Tokyo, Japan: WILEY Blackwell; 2017.

23 Onodera T, Goseki N, Kosaki G. Prognostic nutritional index in gastrointestinal surgery of malnourished cancer patients. Nihon Geka Gakkai Zasshi. 1984 Sep;85(9):1001-5. in Japanese

24 Ying HQ, Deng QW, He BS, Pan YQ, Wang $F$, Sun HL, et al. The prognostic value of preoperative NLR, d-NLR, PLR and LMR for predicting clinical outcome in surgical colorectal cancer patients. Med Oncol. 2014 Dec;31(12):305.

25 World Health Organization. Global database on body mass index. World Health Organaization; 2020.

26 Katayama H, Kurokawa Y, Nakamura K, Ito H, Kanemitsu Y, Masuda N, et al. Extended Clavien-Dindo classification of surgical complications: Japan Clinical Oncology Group postoperative complications criteria. Surg Today. 2016 Jun;46(6):668-85.

27 Liao G, Zhao Z, Yang H, Chen M, Li X. Can prognostic nutritional index be a prediction factor in esophageal cancer? A meta-analysis. Nutr Cancer. 2020;72(2):187-93.

28 Chen S, Yang X, Feng JF. A novel inflammation-based prognostic score for patients with esophageal squamous cell carcinoma: the Creactive protein/prognostic nutritional index ratio. Oncotarget. 2016 Sep 20;7(38):62123-32.

29 Han L, Song Q, Jia Y, Chen X, Wang C, Chen $\mathrm{P}$, et al. The clinical significance of systemic inflammation score in esophageal squamous cell carcinoma. Tumour Biol. 2016 Mar;37(3): 3081-90

30 Ikeguchi M, Kouno Y, Kihara K, Suzuki K, Endo K, Nakamura S, et al. Evaluation of prognostic markers for patients with curatively resected thoracic esophageal squamous cell carcinomas. Mol Clin Oncol. 2016 Dec; 5(6):767-72.

31 Miyazaki T, Sakai M, Sohda M, Tanaka N, Yokobori T, Motegi Y, et al. Prognostic significance of inflammatory and nutritional parameters in patients with esophageal cancer. Anticancer Res. 2016 Dec;36(12):6557-62. 
32 Matsumoto H, Okamoto Y, Kawai A, Ueno D, Kubota H, Murakami H, et al. Prognosis prediction for postoperative esophageal cancer patients using Onodera's prognostic nutritional index. Nutr Cancer. 2017 Aug-Sep; 69(6):849-54.

33 Hirahara N, Tajima Y, Fujii Y, Kaji S, Yamamoto T, Hyakudomi R, et al. Preoperative prognostic nutritional index predicts longterm surgical outcomes in patients with esophageal squamous cell carcinoma. World J Surg. 2018 Jul;42(7):2199-208.

34 Zhang H, Shang X, Ren P, Gong L, Ahmed A, $\mathrm{Ma} Z$, et al. The predictive value of a preoperative systemic immune-inflammation index and prognostic nutritional index in patients with esophageal squamous cell carcinoma. J Cell Physiol. 2019 Feb;234(2):1794-802.

35 McMillan DC. An inflammation-based prognostic score and its role in the nutrition-based management of patients with cancer. Proc Nutr Soc. 2008 Aug;67(3):257-62.

36 Crumley AB, Stuart RC, McKernan M, McMillan DC. Is hypoalbuminemia an independent prognostic factor in patients with gastric cancer? World J Surg. 2010 Oct;34(10): 2393-8.

37 Ligthart-Melis GC, Weijs PJ, te Boveldt ND, Buskermolen S, Earthman CP, Verheul HM, et al. Dietician-delivered intensive nutritional support is associated with a decrease in severe postoperative complications after surgery in patients with esophageal cancer. Dis Esophagus. 2013 Aug;26(6):587-93.

38 Ikeda U, Ohkawa F, Seino Y, Yamamoto K, Hidaka Y, Kasahara T, et al. Serum interleukin 6 levels become elevated in acute myocar- dial infarction. J Mol Cell Cardiol. 1992 Jun; 24(6):579-84

39 Kubota T, Hiki N, Nunobe S, Kumagai K, Aikou S, Watanabe R, et al. Significance of the inflammation-based Glasgow prognostic score for short- and long-term outcomes after curative resection of gastric cancer. J Gastrointest Surg. 2012 Nov;16(11):203744.

40 Hirai T, Yoshimoto A, Iwata T, Yamashita Y, Kuwahara M, Toge T. Enhancing effect of thoraco-laparotomy on liver metastasis and the role played by active oxygens in its mechanism. Surg Today. 1997 Nov 1;27(11): 1040-5.

41 Segatto I, Berton S, Sonego M, Massarut S, Perin T, Piccoli E, et al. Surgery-induced wound response promotes stem-like and tumor-initiating features of breast cancer cells, via STAT3 signaling. Oncotarget. 2014 Aug 15;5(15):6267-79.

42 Nozoe T, Saeki H, Sugimachi K. Significance of preoperative elevation of serum C-reactive protein as an indicator of prognosis in esophageal carcinoma. Am J Surg. 2001 Aug;182(2): 197-201.

43 Shimada H, Nabeya Y, Okazumi S, Matsubara $\mathrm{H}$, Shiratori T, Aoki T, et al. Elevation of preoperative serum C-reactive protein level is related to poor prognosis in esophageal squamous cell carcinoma. J Surg Oncol. 2003 Aug; 83(4):248-52.

44 Gockel I, Dirksen K, Messow CM, Junginger T. Significance of preoperative C-reactive protein as a parameter of the perioperative course and long-term prognosis in squamous cell carcinoma and adenocarcinoma of the oesophagus. World J Gastroenterol. 2006 Jun 21;12(23):3746-50.

45 Saito T, Kurokawa Y, Miyazaki Y, Makino $\mathrm{T}$, Takahashi T, Yamasaki M, et al. Which is a more reliable indicator of survival after gastric cancer surgery: postoperative complication occurrence or C-reactive protein elevation? J Surg Oncol. 2015 Dec;112(8): 894-9.

46 Morita M, Yoshida R, Ikeda K, Egashira A, Oki E, Sadanaga N, et al. Acute lung injury following an esophagectomy for esophageal cancer, with special reference to the clinical factors and cytokine levels of peripheral blood and pleural drainage fluid. Dis Esophagus. 2008;21(1):30-6.

47 Tsujimoto H, Takahata R, Nomura S, Kumano I, Matsumoto Y, Yoshida K, et al. Predictive value of pleural and serum interleukin-6 levels for pneumonia and hypo-oxygenations after esophagectomy. J Surg Res. 2013 Jun 15; 182(2):e61-7.

48 Kamei T, Kitayama J, Yamashita H, Nagawa $\mathrm{H}$. Intraoperative blood loss is a critical risk factor for peritoneal recurrence after curative resection of advanced gastric cancer. World J Surg. 2009 Mar 24;33(6):1240.

49 Liang YX, Guo HH, Deng JY, Wang BG, Ding $\mathrm{XW}$, Wang $\mathrm{XN}$, et al. Impact of intraoperative blood loss on survival after curative resection for gastric cancer. World J Gastroenterol. 2013 Sep 7;19(33):5542-50.

50 Komatsu Y, Orita H, Sakurada M, Maekawa $\mathrm{H}$, Hoppo T, Sato K. Intraoperative blood transfusion contributes to decreased longterm survival of patients with esophageal cancer. World J Surg. 2012 Apr;36(4):844-50. 